\title{
Real-time Control under Clock Offsets between Sensors and Controllers*
}

\author{
Kunihisa Okano ${ }^{\dagger}$ \\ Department of Electrical and \\ Computer Engineering \\ University of California \\ Santa Barbara \\ CA 93106-9560 USA \\ kokano@ece.ucsb.edu
}

\author{
Masashi Wakaiki ${ }^{\ddagger}$ \\ Department of Electrical and \\ Computer Engineering \\ University of California \\ Santa Barbara \\ CA 93106-9560 USA \\ masashiwakaiki \\ @ece.ucsb.edu
}

\author{
João P. Hespanha \\ Department of Electrical and \\ Computer Engineering \\ University of California \\ Santa Barbara \\ CA 93106-9560 USA \\ hespanha@ece.ucsb.edu
}

\begin{abstract}
This paper studies the impact of clock mismatches in spatially distributed real-time control systems. We consider a configuration in which sensor measurements are collected by one processor that transmits the measurements to another control/actuation processor through a network, but the two processors do not have a common clock. Due to the clock mismatch, there will be an offset between the actual time at which a measurement is taken and the time reported by the sensor. Our goal is to discover fundamental limitations to the ability to stabilize the control loop arising from the clocks mismatch. We consider time-varying bounded offsets and derive limitations on the offset bound for the stability of the feedback system. For the case of a scalar linear process, there exists a critical limitation, which depends on the level of instability of the plant and the nominal sampling period. In contrast, for the vector linear processes, if the process dynamics has at least two distinct real eigenvalues, then there is no fundamental limitation on the offset bound.
\end{abstract}

\section{Categories and Subject Descriptors}

C.2.4 [Computer-Communication Networks]: Distributed Systems; I.2.8 [Artificial Intelligence]: Problem Solving, Control Methods, and Search-Control theory

\section{Keywords}

Clock offsets; networked control systems; fundamental limitations.

\footnotetext{
*This material is based upon work supported by the National Science Foundation under Grant No. CNS-1329650.

$\dagger_{\mathrm{K}}$. Okano is supported by JSPS Postdoctoral Fellowships for Research Abroad.

${ }_{\ddagger}^{\ddagger}$ M. Wakaiki acknowledges The Kyoto University Foundation for the support of this work.

Permission to make digital or hard copies of part or all of this work for personal or classroom use is granted without fee provided that copies are not made or distributed for profit or commercial advantage and that copies bear this notice and the full citation on the first page. Copyrights for thirdparty components of this work must be honored. For all other uses, contact the Owner/Author.

Copyright is held by the owner/author(s).

HSCC '15, April 14-16, 2015, Seattle, WA, USA.

ACM 978-1-4503-3433-4/15/04

http://dx.doi.org/10.1145/2728606.2728613
}

\section{INTRODUCTION}

It is widely recognized that control systems can place severe real-time constraints on software systems. However, in the context of distributed control applications, it generally does not suffice to make sure that the different processors involved perform computations in a timely fashion. In fact, a significant challenge in such systems is that the different processors may not share a common notion of time, because their local clocks are not properly synchronized. In this paper, we study the potential impact of clock mismatches in a spatially distributed real-time control system.

We consider control systems where the components are spatially distributed and connected via networks. To pursue real-time control of such systems, one will be required to synchronize the local clocks of the system components [12]. Clock synchronization in distributed systems $[1,6]$ and protocols for synchronization $[10,18,21]$ have been actively studied. In addition to such protocols, information from Global Positioning System (GPS) is often used as a reference of the global clock in practical systems. However, attaining synchronization through communication channels is in general difficult: It has been shown that synchronization is possible only under certain conditions [7]. Moreover, recent works have pointed out that GPS signals can be spoofed and hence are vulnerable against malicious attacks $[2,11]$.

Real-time control has been studied from several view points including providing algorithms for task scheduling $[4,17]$ and dealing with delays and jitters $[3,22]$. However, the fundamental problem that analyzing the impact of clock mismatch on control systems has been an open issue.

In this paper, we consider feedback systems where the sensor measuring the plant state and the controller operate locally and may not be synchronized. Due to this asynchronism, a reported plant state from the sensor may be taken at an instant different from that the controller supposes. This mismatch results in uncertainty in measurements and causes fundamental limitations in control. We consider the situation in which the offset between the clock in the sensor and that in the controller is bounded and may be time varying. This offset is unknown to both the sensor and the controller sides. Our objective is to clarify limitations on the tolerable offsets for stability. For the scalar plants case, we derive a necessary and sufficient condition for stability, which gives a tight upper bound on the offset. In contrast, for the vector case, there exists no practical limitation on the offset if the 
HSCC'15, April 14-16, 2015, Seattle, Washington

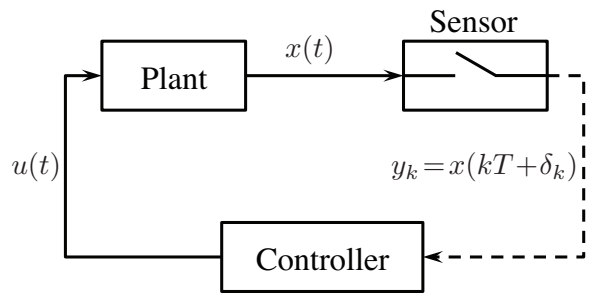

Figure 1: Feedback system.

plant has at least two different real modes.

Systems with variable sampling intervals have been actively studied from the perspective of robust control; see, e.g., $[5,8,13,14]$. In these papers, the controller is commonly given by a linear time-invariant system, where the inputs to the controller are updated at uncertain instants because of the variable sampling period. For the resulting feedback system, its stability against uncertain sampling instants has been analyzed, and sufficient conditions have been shown. Our recent work [20] in this line of research has dealt with constant clock offsets.

Unlike the above-mentioned works, we study the existence of a general causal control algorithm to stabilize the feedback loop in the presence of incorrect timing information. Inspired by the analysis of data rate limitations in control under quantization (see, e.g., $[15,19]$ ), we attempt to quantify how uncertainty in timing information translates into uncertainty in state estimation, under a causality constraint. Stabilization is impossible if the lowest level of uncertainty in state estimation results in an uncertainty set that grows unbounded. Analyzing fundamental limitations due to imperfect measurements in networked control systems has been a vital research area in the last decade. A wide variety of imperfections has been considered including quantization errors due to data rate limitations and packet losses caused by unreliability of channels; see $[9,16]$ and the references therein. In this research, the imperfection is caused by the clock offset between the sensor and the controller.

This paper is organized as follows: In Section 2, we formulate the problem. The definitions of the state estimation sets used to generate the control input are given in Section 3. In Section 4, we consider the case of scalar plants and provide the first main result. Section 5 is devoted to show the result for the vector systems case. In Section 6, a more practical setup is considered where the sensor output is quantized to discrete values. We consider the scalar plants case and generalize the result in Section 4. Finally concluding remarks are given in Section 7 .

Notations: Throughout the paper, $\mathbb{Z}_{+}$denotes the set of nonnegative integers; 0 (bold-faced zero) stands for the zero vector of an appropriate dimension; $o(\cdot)$ is the Landau symbol defined as $f=o(\phi)$ means that $f / \phi \rightarrow 0$; and $\|\cdot\|$ represents the Euclidean norm.

\section{PROBLEM FORMULATION}

Consider the feedback system depicted in Figure 1. The plant system to be controlled is the following continuoustime linear time-invariant process:

$$
\dot{x}(t)=A x(t)+B u(t), \quad x(0) \in \mathbb{R}^{n}, \quad t \geq 0,
$$

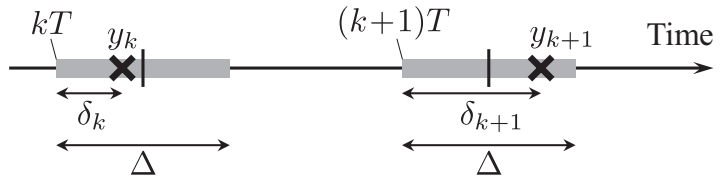

Figure 2: Time chart.

where $x(t) \in \mathbb{R}^{n}$ is the state and $u(t) \in \mathbb{R}^{m}$ is the input. The unknown initial state $x(0)$ may be anywhere in $\mathbb{R}^{n}$. We introduce the following assumption on the plant.

Assumption 1. The system (1) is unstable and controllable, and $A$ is invertible.

The plant state is periodically observed by a sensor and is sent to the controller. Let $y_{k}, k \in \mathbb{Z}_{+}$, be the $k$ th observed state, and let $T>0$ be the sampling period from the perspective of the controller clock. Figure 2 illustrates a time chart of sampling: The actual time instants at which the samples $y_{k}$ are generated are represented by the crosses, whereas the gray boxes represent the uncertainty inherent to the timing information. due to the (variable) offset between the clocks of the sensor and the controller. We assume that the magnitude of the clock offset is bounded by $\Delta / 2$. The offset bound $\Delta$ is smaller than the sampling interval $T$ :

$$
0 \leq \Delta<T \text {. }
$$

There are various algorithms for the clock offset estimation, and the resulted estimation errors have been analyzed; see, e.g., [21]. These results are useful to determine the offset bound $\Delta$. We denote by $k T+\Delta / 2$ the $k$ th nominal sampling instant (center of the timing uncertainty boxes in Figure 2). Then, $y_{k}$ is given by

$$
y_{k}=x\left(k T+\delta_{k}\right), \quad \delta_{k} \in[0, \Delta] .
$$

Here, the actual sampling instant $k T+\delta_{k}$ according to the controller clock is unknown because of the unknown jitter $\delta_{k}$. We note that the controller cannot determine $\delta_{k}$ from the time instant at which the sample $y_{k}$ arrives since there may exist an unknown communication delay.

Based on the received observations $y_{0}, \ldots, y_{k}$, the controller determines the input $u(t), t>k T+\Delta$. We make the following assumption.

Assumption 2. The control input is constant during $[k T$, $k T+\Delta]$ for every $k$ :

$$
u(t)=u_{k}, \quad \forall t \in[k T, k T+\Delta], \quad \forall k \in \mathbb{Z}_{+} .
$$

This assumption implies that during the intervals in which the sensor may measure the plant state, the controller holds the input. For the inputs during $t \in(k T+\Delta,(k+1) T)$, we allow the controller to take any value.

REMARK 1. Assumption 2 is required to prove the necessity results in the paper. We note that the sufficient conditions for stability hold even if we limit the class of controllers to piecewise constant ones.

Our objective is to clarify how large $\Delta$ can be to ensure the stabilizability of the system in the following sense.

Definition 1. The feedback system is stabilizable if for any sequence of jitters $\left\{\delta_{k}\right\}_{k=0}^{\infty}, \delta_{k} \in[0, \Delta]$, and any initial state $x(0)$, there exists a controller such that the state converges to the origin, i.e., $\lim _{t \rightarrow \infty} x(t)=0$. 


\section{COMPUTATION OF ESTIMATION SETS}

In what follows, we present notations and definitions of estimation sets of the plant states, which will be referred to in the following sections. Let $I_{k}^{-}$be the initial estimation set of $x(k T)$ based on the past observations $y_{0}, \ldots, y_{k-1}$. From (3), $y_{k}$ satisfies

$$
y_{k}=\mathrm{e}^{A \delta_{k}} x(k T)+\int_{k T}^{k T+\delta_{k}} \mathrm{e}^{A\left(k T+\delta_{k}-\tau\right)} B u(\tau) \mathrm{d} \tau
$$

with the unknown parameter $\delta_{k}$ and the true state $x(k T)$. Thus, $x(k T)$ must lie in the following set $J_{k}$ :

$$
\begin{aligned}
J_{k} & =\left\{\mathrm{e}^{-A \delta_{k}} y_{k}-\int_{k T}^{k T+\delta_{k}} \mathrm{e}^{A(k T-\tau)} B u(\tau) \mathrm{d} \tau: \delta_{k} \in[0, \Delta]\right\} \\
& =\left\{\mathrm{e}^{-A \delta_{k}}\left(y_{k}+A^{-1} B u_{k}\right)-A^{-1} B u_{k}: \delta_{k} \in[0, \Delta]\right\} .
\end{aligned}
$$

Here, the second equality follows by Assumption 2. With this estimation set $J_{k}$, after receiving $y_{k}$, we obtain the updated estimation set $I_{k}$ as

$$
I_{k}:=I_{k}^{-} \cap J_{k} .
$$

The initial estimation set of $x((k+1) T)$ is computed using $I_{k}$ by

$I_{k+1}^{-}=\left\{\mathrm{e}^{A T} \hat{x}_{k}+\int_{k T}^{(k+1) T} \mathrm{e}^{A((k+1) T-\tau)} B u(\tau) \mathrm{d} \tau: \hat{x}_{k} \in I_{k}\right\}$.

Since $I_{0}^{-}=\mathbb{R}^{n}$ from the setup, we have $I_{0}=J_{0}$ for any $J_{0}$. By (8), the initial estimation set of $x(T)$ is

$$
I_{1}^{-}=\left\{\mathrm{e}^{-A \delta_{0}} a_{0}+b_{0}: \delta_{0} \in[0, \Delta]\right\}
$$

where

$$
\begin{aligned}
& a_{0}:=\mathrm{e}^{A T}\left(y_{0}+A^{-1} B u_{0}\right), \\
& b_{0}:=-\mathrm{e}^{A T} A^{-1} B u_{0}+\int_{0}^{T} \mathrm{e}^{A(T-\tau)} B u(\tau) \mathrm{d} \tau .
\end{aligned}
$$

After receiving $y_{1}$, we obtain the new estimation set

$$
J_{1}=\left\{\mathrm{e}^{-A \delta_{1}} a_{1}+b_{1}: \delta_{1} \in[0, \Delta]\right\},
$$

where $a_{1}:=y_{1}+A^{-1} B u_{1}$ and $b_{1}:=-A^{-1} B u_{1}$.

We note that the set $I_{k}$ given in (7) is a tight estimation set of $x(k T)$ in the following sense: For any $\hat{x}_{k} \in I_{k}$, there exists a path of the state $x(t), t \in[0, k T+\Delta]$, which results in $x(k T)=\hat{x}_{k}$ and corresponds to the observed outputs $y_{0}, y_{1} \ldots, y_{k}$ with possible delays $\left\{\delta_{i}\right\}_{i=0}^{k}$. We formally define tightness of an estimation set below.

Definition 2. For an estimation set $I_{k} \subset \mathbb{R}^{n}$ of $x(k T)$, which is constructed from $I_{0}^{-}, u(t), t \in[0, k T+\Delta]$, and $y_{0}, y_{1}, \ldots, y_{k}$, the estimation set is said to be tight if for every $\hat{x}_{k} \in I_{k}$, there exist $x_{0} \in I_{0}^{-}$and $\left\{\delta_{i}\right\}_{i=0}^{k}, \delta_{i} \in[0, \Delta]$, such that

$$
\begin{aligned}
& \mathrm{e}^{A k T} x_{0}+\int_{0}^{k T} \mathrm{e}^{A(k T-\tau)} B u(\tau) \mathrm{d} \tau=\hat{x}_{k}, \\
& \mathrm{e}^{A\left(i T+\delta_{i}\right)} x_{0}+\int_{0}^{i T+\delta_{i}} \mathrm{e}^{A\left(i T+\delta_{i}-\tau\right)} B u(\tau) \mathrm{d} \tau=y_{i}, \forall i .
\end{aligned}
$$

Proposition 1. The estimation set $I_{k}$ defined in $(7)$ is tight in the sense given in Definition 2.
Proof. It is obvious from (5) that $I_{0}=J_{0}$ is tight. Suppose that $I_{k-1}, k \geq 1$, is tight. That is, for any $\hat{x}_{k-1} \in I_{k-1}$, there exist $x_{0} \in I_{0}^{-}$and $\left\{\delta_{i}\right\}_{i=0}^{k-1}, \delta_{i} \in[0, \Delta]$, such that

$$
\begin{aligned}
& \mathrm{e}^{A(k-1) T} x_{0}+\int_{0}^{(k-1) T} \mathrm{e}^{A((k-1) T-\tau)} B u(\tau) \mathrm{d} \tau=\hat{x}_{k-1}, \\
& \mathrm{e}^{A\left(i T+\delta_{i}\right)} x_{0}+\int_{0}^{i T+\delta_{i}} \mathrm{e}^{A\left(i T+\delta_{i}-\tau\right)} B u(\tau) \mathrm{d} \tau=y_{i}, \\
& i=0,1, \ldots, k-1 .
\end{aligned}
$$

Now pick any $\hat{x}_{k}$ from $I_{k}$. Since $\hat{x}_{k} \in I_{k}^{-}$and by (8), there exists $\hat{x}_{k-1} \in I_{k-1}$ such that

$$
\mathrm{e}^{A T} \hat{x}_{k-1}+\int_{(k-1) T}^{k T} \mathrm{e}^{A(k T-\tau)} B u(\tau) \mathrm{d} \tau=\hat{x}_{k} .
$$

Substituting (13) into the above equation, we have that (11) holds with $x_{0}$ selected in (13). Furthermore, with the fact that $\hat{x}_{k} \in J_{k}$ and (5), there exists $\delta_{k} \in[0, \Delta]$ satisfying

$$
\mathrm{e}^{A \delta_{k}} \hat{x}_{k}+\int_{k T}^{k T+\delta_{k}} \mathrm{e}^{A\left(k T+\delta_{k}-\tau\right)} B u(\tau) \mathrm{d} \tau=y_{k} .
$$

We substitute (11) into the above equation and then obtain (12) for the case that $i=k$, which concludes the proof.

\section{LIMITATION ON CLOCK OFFSET: SIN- GLE EIGENVALUE CASE}

In this section, we consider the scalar plants case and derive a tight bound on the maximum offset $\Delta$ for stability. Consider the following system:

$$
\dot{x}(t)=\lambda x(t)+u(t), \quad x(0) \in \mathbb{R} .
$$

In the above system, from the controllability, we have assumed $B=1$ without loss of generality. Moreover, from the instability it follows that $\lambda>0$.

The following is the first main result.

THEOREM 1. The feedback system with the scalar plant (14) is stabilizable in the sense of Definition 1 if and only if the bound on the offset satisfies $0 \leq \Delta<\bar{\Delta}$, where

$$
\bar{\Delta}:= \begin{cases}T & \text { if } 0<T \leq \frac{1}{\lambda} \ln 3 \\ T-\frac{1}{\lambda} \ln \left(\mathrm{e}^{\lambda T}-2\right) & \text { if } T>\frac{1}{\lambda} \ln 3 .\end{cases}
$$

We note that the two expressions in (15) are continuous. The limitation $\bar{\Delta}=T$ for the case $0<T<(\ln 3) / \lambda$ is due to the assumption (2). The bound $T-\left\{\ln \left(\mathrm{e}^{\lambda T}-2\right)\right\} / \lambda$ is decreasing with respect to $\lambda$ and $T$ when $T>(\ln 2) / \lambda$. This observation is consistent with the intuition that larger evolution rate $\mathrm{e}^{\lambda T}$ per sampling period will result in higher requirement in accuracy of the observations $y_{k}$. The following example shows how the bound $\bar{\Delta}$ varies with $T$.

Example 1. Consider the plant where $\lambda=1.0$. In Figure 3 , we plot the limitation $\bar{\Delta}$ versus the sampling period $T$. The solid line is the active bound $\bar{\Delta}$ and the vertical dotted lines indicate $T=(\ln 2) / \lambda($ left $)$ and $T=(\ln 3) / \lambda$ (right), respectively. For small $T$, the restriction due to (2) limits $\bar{\Delta}$ as $T$. At $T=(\ln 3) / \lambda, \bar{\Delta}$ takes its maximum and the two expressions in (15) coincide with each other. 


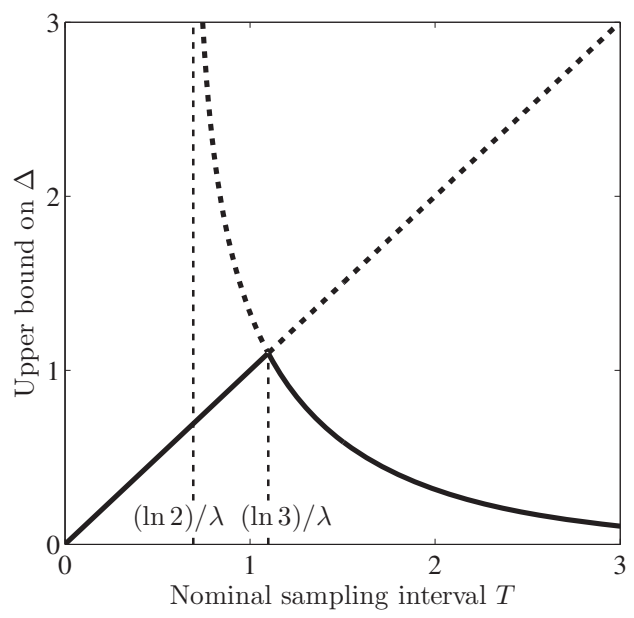

Figure 3: Upper bound on $\Delta$ versus sampling period $T(\lambda=1.0)$ : The solid line is the active bound $\bar{\Delta}$ and the vertical dotted lines indicate $T=(\ln 2) / \lambda$ (left) and $T=(\ln 3) / \lambda$ (right), respectively.

\subsection{Proof of Theorem 1}

For scalar plants, by the definition (6), the estimation set $J_{k}$ computed from $y_{k}$ is written as

$$
J_{k}=\left\{\mathrm{e}^{-\lambda \delta_{k}}\left(y_{k}+\lambda^{-1} u_{k}\right)-\lambda^{-1} u_{k}: \delta_{k} \in[0, \Delta]\right\} .
$$

Thus, $J_{k}$ becomes a closed interval in $\mathbb{R}$. Therefore, with (7) and (8), $I_{k}$ and $I_{k+1}^{-}, k \in \mathbb{Z}_{+}$, also result in intervals in $\mathbb{R}$. Notice that we have assumed $I_{0}^{-}=\mathbb{R}$.

Let $l_{k}$ be the length of the interval $I_{k}$, which is the updated estimation set of $x(k T)$. It is clear that if $l_{k} \rightarrow 0$ as $k \rightarrow$ $\infty$ then the feedback system is stabilizable. We also have that since $I_{k}$ is a tight estimation set, if $\lim _{k \rightarrow \infty} l_{k} \neq 0$, then there exists a possible trajectory of $x(t)$ which does not converge to the origin no matter what we choose for $u(t)$. In the following, we consider a condition that $l_{k} \rightarrow 0$ to show the theorem. Suppose that $I_{k-1}$ is given at $t=$ $(k-1) T+\Delta$. Then one can compute $I_{k}^{-}$from $(8)$. The length $l_{k}$ is determined by $I_{k}^{-}$and $J_{k}$, that is, $l_{k}$ depends on the true state $x_{k} \in I_{k}^{-}$, the jitter $\delta_{k} \in[0, \Delta]$, and the input $u(t), t \in((k-1) T+\Delta, k T)$. To guarantee stability for all possible $x_{k}$ and $\delta_{k}$, we have to care the worst case. Hence, we need a condition for

$$
\max _{x_{k} \in I_{k}^{-}, \delta_{k} \in[0, \Delta]} l_{k} \rightarrow 0 \text { as } k \rightarrow \infty .
$$

In addition, for the necessity part of the theorem, we pursue a condition guaranteeing

$$
\min _{u(t), t \in((k-1) T+\Delta, k T)} \max _{x_{k} \in I_{k}^{-}, \delta_{k} \in[0, \Delta]} l_{k} \rightarrow 0 \text { as } k \rightarrow \infty .
$$

The following lemma gives the expansion rate of $l_{k}$.

Lemma 1. Given an interval $I_{k-1}, k \geq 1$. It follows that

$$
\begin{array}{r}
\min _{u(t), t \in((k-1) T+\Delta, k T)} \max _{x_{k} \in I_{k}^{-}, \delta_{k} \in[0, \Delta]} l_{k} \\
=\frac{\left(1-\mathrm{e}^{-\lambda \Delta}\right) \mathrm{e}^{\lambda T}}{2} l_{k-1} .
\end{array}
$$

Proof. For simplicity of notation in the proof, we first introduce biased sets as follows: Define $\tilde{y}:=y_{k}+\lambda^{-1} u_{k}$ and let $\tilde{J}:=\left\{z+\lambda^{-1} u_{k}: z \in J_{k}\right\}$. Then, from (16), this biased estimation set $\tilde{J}$ can be simply represented as

$$
\tilde{J}= \begin{cases}{\left[\mathrm{e}^{-\lambda \Delta} \tilde{y}, \tilde{y}\right]} & \text { if } \tilde{y}>0 \\ \{0\} & \text { if } \tilde{y}=0 \\ {\left[\tilde{y}, \mathrm{e}^{-\lambda \Delta} \tilde{y}\right]} & \text { if } \tilde{y}<0\end{cases}
$$

Similarly, define the biased sets

$$
\begin{aligned}
& \tilde{I}^{-}:=\left\{z+\lambda^{-1} u_{k}: z \in I_{k}^{-}\right\}, \\
& \tilde{I}:=\tilde{I}^{-} \cap \tilde{J}=\left\{z+\lambda^{-1} u_{k}: z \in I_{k}\right\} .
\end{aligned}
$$

Note that the length $l_{k}$ of $I_{k}$ is the same as that of $\tilde{I}$ and the analysis in this proof holds for any bias $\lambda^{-1} u_{k}$. In Figure 4, we illustrate the update procedure of the estimation set.

We now show that the input $u(t), t \in((k-1) T+\Delta, k T)$, achieving the minimum in (17) is the one which makes $\tilde{I}^{-}$ symmetric about the origin. In what follows, we will evaluate the worst case length $\max _{x_{k}, \delta_{k}} l_{k}$ for a given $\tilde{I}^{-}$. We start by describing the range of $y_{k}$ under the knowledge that $x_{k} \in I_{k}^{-}$and $\delta_{k} \in[0, \Delta]$. With (4), the set of all possible $y_{k}$ is as follows:

$$
y_{k} \in\left\{\mathrm{e}^{\lambda \delta_{k}} x_{k}+\lambda^{-1}\left(\mathrm{e}^{\lambda \delta_{k}}-1\right) u_{k}: x_{k} \in I_{k}^{-}, \delta_{k} \in[0, \Delta]\right\},
$$

which results in

$$
\tilde{y} \in\left\{\mathrm{e}^{\lambda \delta_{k}} z: \delta_{k} \in[0, \Delta], z \in \tilde{I}^{-}\right\} .
$$

Thus, we can rewrite the maximization term in (17) as

$$
\max _{x_{k} \in I_{k}^{-}, \delta_{k} \in[0, \Delta]} l_{k}=\max _{\tilde{y} \in\left\{\mathrm{e}^{\lambda \delta_{k}} z: \delta_{k} \in[0, \Delta], z \in \tilde{I}^{-}\right\}} l_{k} .
$$

Next, we evaluate $\max _{\tilde{y}} l_{k}$ for each variation of $\tilde{I}^{-}$. In the rest of the proof, we assume $\tilde{y} \neq 0$ since by (18), it is clear that $\tilde{y}=0$ does not maximize $l_{k}$. Let denote the lower bound and the upper bound of $\tilde{I}^{-}$by $p$ and $q$, respectively: $\tilde{I}^{-}=[p, q]$. Suppose that

$$
|q| \geq|p|
$$

i.e., the midpoint of $\tilde{I}^{-}$is nonnegative. For the case $|q|<|p|$, one can apply the same discussion below by flipping signs.

Consider the following two cases (i) $p \leq 0$, that is, $\tilde{I}^{-}$ contains the origin and (ii) $p>0, \tilde{I}^{-}$lies only in the positive area.

(i) $p \leq 0$ : In this case, it follows that $q \geq 0$ from (20). By (18), we have

$$
\tilde{I}= \begin{cases}{\left[\mathrm{e}^{-\lambda \Delta} \tilde{y}, \min (q, \tilde{y})\right]} & \text { if } \tilde{y}>0 \\ {\left[\max (p, \tilde{y}), \mathrm{e}^{-\lambda \Delta} \tilde{y}\right]} & \text { if } \tilde{y}<0\end{cases}
$$

Taking maximums in both cases $\tilde{y}>0$ and $\tilde{y}<0$, and using (20), one can see that when $\tilde{y}=q$ the length $l_{k}$ takes its maximum

$$
\max _{\tilde{y} \in\left\{\mathrm{e}^{\lambda \delta_{k}} z: \delta_{k} \in[0, \Delta], z \in \tilde{I}^{-}\right\}} l_{k}=\left(1-\mathrm{e}^{-\lambda \Delta}\right) q .
$$

The right-hand side takes its minimum under the conditions (20) and (i) for the case in which $|q|=|p|$ or $\tilde{I}^{-}$is symmetry about the origin. 


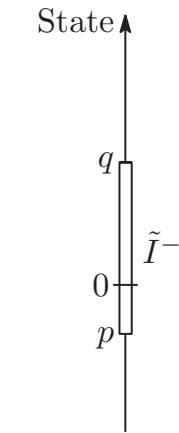

Time $k T$

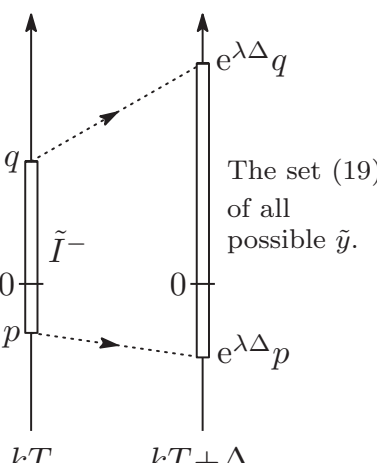

$k T \quad k T+\Delta$

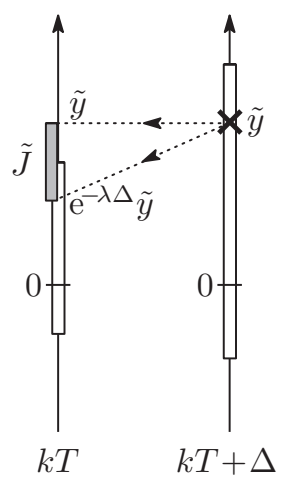

(c)

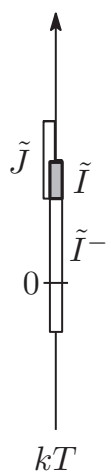

(d)

Figure 4: Update procedure of the estimation set: (a) The initial estimation set $\tilde{I}^{-}$is depicted as the white box; (b) $\tilde{I}^{-}$expands during the sensing interval $[k T, k T+\Delta]$; (c) After receiving $\tilde{y}$, the controller computes the set $\tilde{J}$ of the candidates of the state at $k T$ which may result in $\tilde{y}$ somewhere in $[k T, k T+\Delta]$; (d) The updated estimation set $\tilde{I}$ is obtained as the intersection of $\tilde{I}^{-}$and $\tilde{J}$.

(ii) $p>0$ : In this case, noticing that all possible $\tilde{y}$ are positive from (19), we obtain

$$
\tilde{I}=\left[\max \left(p, \mathrm{e}^{-\lambda \Delta} \tilde{y}\right), \min (q, \tilde{y})\right] .
$$

Therefore,

$$
\max _{\tilde{y} \in\left\{\mathrm{e}^{\lambda \delta} k z: \delta_{k} \in[0, \Delta], z \in \tilde{I}^{-}\right\}} l_{k}= \begin{cases}\left(1-\mathrm{e}^{-\lambda \Delta}\right) q & \text { if } q \geq \mathrm{e}^{\lambda \Delta} p, \\ q-p & \text { if } q<\mathrm{e}^{\lambda \Delta} p .\end{cases}
$$

Note that $q-p$ in the right-hand side does not vary with the position of $\tilde{I}^{-}$. The other expression of $\max _{\tilde{y}} l_{k},\left(1-\mathrm{e}^{-\lambda \Delta}\right) q$, is the same formula for the case (i) as in (21). However, from the conditions (i) and (ii), it is obvious that $q$ cannot be small as that for the case (i). Thus, $\tilde{I}^{-}$satisfying (ii) cannot minimize $\max _{\tilde{y}} l_{k}$.

From the discussions (i) and (ii), we have that $\max _{\tilde{y}} l_{k}$ is minimized when $\tilde{I}^{-}$is taken as satisfying $|q|=|p|=$ $\mathrm{e}^{\lambda T} l_{k-1} / 2$, and the minimum is

$$
\min _{u(t), t \in((k-1) T+\Delta, k T)} \max _{x_{k} \in I_{k}^{-}, \delta_{k} \in[0, \Delta]} l_{k}=\left(1-\mathrm{e}^{-\lambda \Delta}\right) \frac{\mathrm{e}^{\lambda T}}{2} l_{k-1} .
$$

This concludes the proof.

Proof of Theorem 1. From Lemma 1, the system (14) is stable if and only if

$$
\frac{\left(1-\mathrm{e}^{-\lambda \Delta}\right) \mathrm{e}^{\lambda T}}{2}<1 .
$$

From this inequality and the assumption (2), we have the upper bound (15) on $\Delta$.

We now present a numerical example to illustrate behavior of the system.

Example 2. Consider the system where the plant parameter is $\lambda=1.0$ and the nominal sampling period is $T=2.0$. We employ the following control law: Divide the interval $((k-1) T+\Delta, k T)$ into two parts of the same length. In the first time period, the controller applies the constant input which makes $I_{k}^{-}$symmetric about $\lambda^{-1} u_{k}$. As we have seen in the proof of Lemma 1, this control input minimizes $\max _{x_{k}, \delta_{k}} l_{k}$, which is the length of the estimation set $I_{k}$ in the worst case. The input in the later period and $u_{k}$ are taken to be 0 . We take the initial state as $x(0)=1$ and chose $\delta_{k}$ uniformly randomly from $[0, \Delta]$. Note that these variables are unknown to the controller. We first consider the case that the offset bound is small and satisfies the stability condition in Theorem 1 ; suppose $\Delta=0.30$, which is less than $\bar{\Delta} \approx 0.32$. In Figure $5 \mathrm{a}$, we illustrate the state $x(t)$ and the input $u(t)$ by the solid line and the dashed line, respectively. Moreover, the observations $y_{k}$ are depicted by the cross marks and the upper bounds and the lower bounds of the estimation sets $I_{k}$ are represented by $\Delta$ and $\nabla$, respectively. One can observe that as time progresses, the upper bounds and the lower bounds of $I_{k}$ become closer, and the state successfully converges to 0 . Next, consider the case $\Delta=0.45$, which violates the stability limit $\bar{\Delta} \approx 0.32$. Figure $5 \mathrm{~b}$ shows the behavior of the system in this case. We see that the state (solid line) oscillates and the estimation set $I_{k}$ (triangle marks) does not shrink to a single point.

\section{LIMITATION ON CLOCK OFFSET: MULTIPLE EIGENVALUES CASE}

In this section, we consider plants with multi eigenvalues and study the maximum offset tolerable to achieve stability.

The following theorem is the main result in this section.

THEOREM 2. If $A$ has at least two different real eigenvalues, then for any $\Delta$ satisfying (2), the state of the feedback system with (1) can be reconstructed in finite steps.

From this theorem and the controllability, the stabilizability result follows immediately.

Corollary 1. If $A$ has at least two different real eigenvalues, then for any $\Delta$ satisfying (2), the feedback system with (1) is stabilizable in the sense of Definition 1 in finite steps.

Therefore, unlike the scalar case, there is no practical limitation on the offset if the plant dynamics has two different real eigenvalues. We may roughly explain this result as follows: At the time after receiving $y_{k}=x\left(k T+\delta_{k}\right)$, there are 


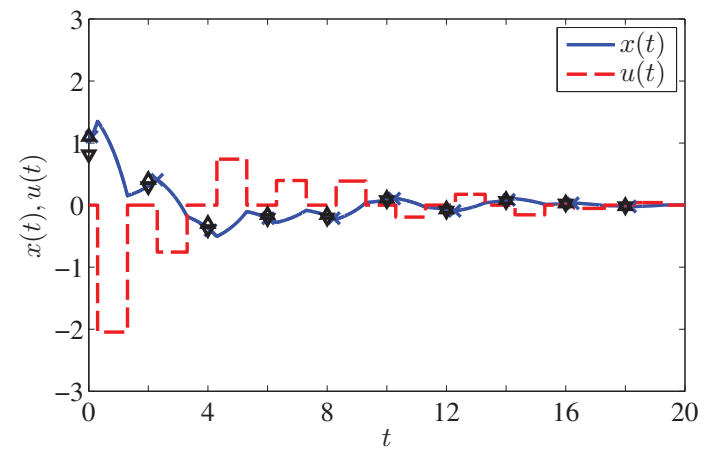

(a) $\Delta=0.30<\bar{\Delta} \approx 0.32$

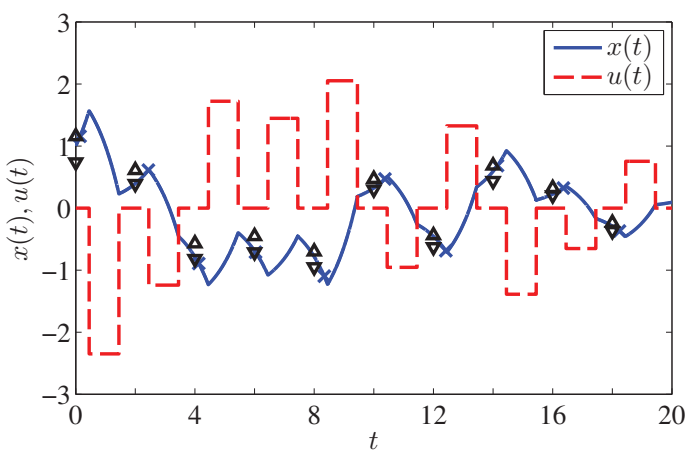

(b) $\Delta=0.45>\bar{\Delta} \approx 0.32$

Figure 5: Behavior of the system $(\lambda=1.0, T=2.0$, $\bar{\Delta} \approx 0.32$ ): The plant state $x(t)$ (solid), the input $u(t)$ (dashed), the observations $y_{k}$ (cross), and the estimation sets $I_{k}$ ( $\triangle$ : upper bounds, $\nabla$ : lower bounds).

$n+k+1$ unknown variables, i.e., $x(0)$ and $\delta_{0}, \ldots, \delta_{k}$. On the other hand, if $A$ has two different eigenvalues, we have $2(k+1)$ independent equations from evolution of the states corresponding to the eigenvalues. Hence, one can determine the exact state in finite steps. Moreover, since the plant is controllable, there exists an input which drives the state to the origin in a finite time interval.

The proof of Theorem 2 consists of two steps. We first consider a subsystem of (1). Since there exist eigenvalues $\lambda_{1}, \lambda_{2} \in \mathbb{R}$ of $A$ such that $\lambda_{1} \neq \lambda_{2}$, via an appropriate coordinate transformation, we have

$$
V^{-1} \dot{x}(t)=\left[\begin{array}{lll}
\lambda_{1} & & \\
& \lambda_{2} & \\
& & *
\end{array}\right] V^{-1} x(t)+V^{-1} B u(t),
$$

for some matrix $V \in \mathbb{R}^{n \times n}$. Extracting the first two rows of the above system, we obtain the subsystem

$$
\dot{\xi}(t)=\Lambda \xi(t)+\nu(t)
$$

where $\Lambda:=\operatorname{diag}\left(\lambda_{1}, \lambda_{2}\right)$, and $\xi$ and $\nu$ are the corresponding states and the inputs, respectively.

As the first step of the proof, we consider computing estimation sets of $\xi(k T)$ from $y_{0}, \ldots, y_{k}$. Instead of the estimation sets of $x$ represented by $J_{k}, I_{k}$, and $I_{k+1}^{-}$, which are defined in (6)-(8), we denote the corresponding estimation sets of $\xi$ by $\hat{J}_{k}, \hat{I}_{k}$, and $\hat{I}_{k+1}^{-}$. In light of (9), the estimation set $\hat{I}_{1}^{-}$of $\xi(T)$ is expressed as

$$
\hat{I}_{1}^{-}=\left\{\mathrm{e}^{-\Lambda \delta_{0}} \hat{a}_{0}+\hat{b}_{0}: \delta_{0} \in[0, \Delta]\right\} .
$$

Here, $\hat{a}_{0}$ and $\hat{b}_{0}$ are defined as follows: Denote by $\zeta_{k}$ the first two elements of $V^{-1} y_{k}$, and let $\nu_{k}$ and $\nu(t)$ be the first two elements of $V^{-1} B u_{k}$ and $V^{-1} B u(t)$, respectively. Then, $\hat{a}_{0}$ and $\hat{b}_{0}$ are defined as

$$
\begin{aligned}
& \hat{a}_{0}:=\mathrm{e}^{\Lambda T}\left(\zeta_{0}+\Lambda^{-1} \nu_{0}\right), \\
& \hat{b}_{0}:=-\mathrm{e}^{\Lambda T} \Lambda^{-1} \nu_{0}+\int_{0}^{T} \mathrm{e}^{\Lambda(T-\tau)} \nu(\tau) \mathrm{d} \tau .
\end{aligned}
$$

Note that $\Lambda$ is nonsingular since $A$ is invertible. Moreover, by (10), it follows that

$$
\begin{aligned}
& \hat{J}_{1}=\left\{\mathrm{e}^{-\Lambda \delta_{1}} \hat{a}_{1}+\hat{b}_{1}: \delta_{1} \in[0, \Delta]\right\}, \\
& \text { where } \quad \hat{a}_{1}:=\zeta_{1}+\Lambda^{-1} \nu_{1}, \quad \hat{b}_{1}:=-\Lambda^{-1} \nu_{1} .
\end{aligned}
$$

We claim that the intersection of $\hat{I}_{1}^{-}$and $\hat{J}_{1}$, which is the updated estimation set $\hat{I}_{1}$, can be a set containing only finite elements. This claim is formally stated in the following lemma.

Lemma 2. There exists a control input $\nu(t), t \in[0, T+\Delta]$ that makes the updated estimation set $\hat{I}_{1}=\hat{I}_{1}^{-} \cap \hat{J}_{1}$ a set of finite points.

To prove this lemma, we will need to consider the following problem: Let $a, b$, and $c$ be nonzero vectors in $\mathbb{R}^{2}$; also fix $\left[T_{1}, T_{2}\right] \subset \mathbb{R}$ and $\left[T_{3}, T_{4}\right] \subset \mathbb{R}$. Define functions $f:\left[T_{1}, T_{2}\right] \rightarrow$ $\mathbb{R}^{2}$ and $g:\left[T_{3}, T_{4}\right] \rightarrow \mathbb{R}^{2}$ as

$$
f(t):=\mathrm{e}^{\Lambda t} a, \quad g(t):=\mathrm{e}^{\Lambda t} b+c .
$$

Then, find the intersection of $\left\{f(t): t \in\left[T_{1}, T_{2}\right]\right\}$ and $\{g(t)$ : $\left.t \in\left[T_{3}, T_{4}\right]\right\}$.

The following lemma states that the intersection can be a set of finite points if $a, b$, and $c$ are chosen appropriately.

LEMMA 3. If at least one of the vectors $a, b$, and $c$ is not an eigenvector of $\Lambda$, then the intersection of $\{f(t): t \in$ $\left.\left[T_{1}, T_{2}\right]\right\}$ and $\left\{g(t): t \in\left[T_{3}, T_{4}\right]\right\}$ becomes a set of finite points.

Proof. See Section 5.1.

We now prove Lemma 2 using Lemma 3.

Proof of Lemma 2. Consider the statement of Lemma 3 with $a=\hat{a}_{0}, b=\hat{a}_{1}$, and $c=\hat{b}_{0}-\hat{b}_{1}$. Then, $\hat{I}_{1}$ corresponds to the intersection of $\left\{f(t): t \in\left[T_{1}, T_{2}\right]\right\}$ and $\{g(t): t \in$ $\left.\left[T_{3}, T_{4}\right]\right\}$. Note that $\hat{a}_{0}$ and $\hat{a}_{1}$ can be assumed to be nonzero since if either $\hat{a}_{0}=\mathbf{0}$ or $\hat{a}_{1}=\mathbf{0}$, then $\hat{I}_{1}^{-}$or $\hat{J}_{1}$ becomes a single point. Thus, such cases can be excluded.

Moreover, in what follows, we show that there exists an input to make $\hat{b}_{0}-\hat{b}_{1}$ not to be $\mathbf{0}$ nor an eigenvector of $\Lambda$. Since $\nu(t)=\nu_{0}, t \in[0, \Delta]$, it follows from (24) and (24) that

$$
\hat{b}_{0}-\hat{b}_{1}=-\Lambda^{-1} \mathrm{e}^{\Lambda(T-\Delta)} \nu_{0}+\int_{\Delta}^{T} \mathrm{e}^{\Lambda(T-\tau)} \nu(\tau) \mathrm{d} \tau+\Lambda^{-1} \nu_{1} .
$$

Note that the first and the third terms in the right-hand side are known to the controller. Furthermore, the integral term is the zero-state-response of (22). Because of the controllability of the original system (1), $x(k T)$ or $\xi(k T)$ can be set arbitrarily, and hence $\hat{b}_{0}-\hat{b}_{1}$ can be an arbitrary vector 
with the appropriate input $\nu(t), t \in(\Delta, T)$. Thus, with an input $\nu(t)$ such that $\hat{b}_{0}-\hat{b}_{1}$ is not $\mathbf{0}$ nor an eigenvector of $\Lambda$, from Lemma $3, \hat{I}_{1}$ becomes a set of finite points.

The second step, which completes the proof of Theorem 2 , is to provide a procedure to determine the state using $\hat{I}_{1}$ consisting of finite points.

Proof of Theorem 2. Since each point of $\hat{I}_{1}$ corresponds to a candidate of the sequences of past offsets $\delta_{0}, \delta_{1}$, we can determine the estimations of $x(T)$ or $I_{1}$ as a set of finite points. From (8), the initial estimation set $I_{2}^{-}$of $x(2 T)$ also results in finite point. Here, the number of the elements of $I_{2}^{-}$is the same as that of $I_{1}$. Thus, $I_{2}$ become also finite points and its number of the elements is less than or equal to that of $I_{2}^{-}$.

On the other hand, from the controllability, the controller can bring any point in $I_{2}$ to the origin by an appropriate input $u(t), t \in(2 T+\Delta, 3 T)$. Hence, we can reduce the number of the elements of $I_{k}$ at least by one per sampling interval. Repeating this procedure, we have $I_{k}$ as a single point in finite steps.

We now show an example where $I_{1}$ becomes a single point. In this case, we can stabilize the system using only two observations $y_{0}$ and $y_{1}$.

Example 3. Consider the following system:

$$
A=\left[\begin{array}{ll}
2 & 0 \\
0 & 1
\end{array}\right], \quad B=\left[\begin{array}{l}
1 \\
1
\end{array}\right], \quad T=1.5, \quad \Delta=1.0 .
$$

Notice that $\Delta$ is greater than the bound (15) in the scalar case corresponding to each eigenvalue, 2 and 1 , of $A$ since $T-\left\{\ln \left(\mathrm{e}^{2 T}-2\right)\right\} / 2 \approx 0.052$ and $T-\ln \left(\mathrm{e}^{T}-2\right) \approx 0.59$. In Figure 6 , we plot the estimation sets $I_{0}, I_{1}^{-}$, and $J_{1}$ computed from $y_{0}$ and $y_{1}$ in the state space $\mathbb{R}^{2}$. Since we assume $I_{0}^{-}=\mathbb{R}^{2}, I_{0}$ is equal to $J_{0}$. Note that the unknown state $x(0)$ and $x(T)$ are not used to compute the estimation sets. The figure shows that $I_{1}^{-} \cap J_{1}=I_{1}$ becomes a single point, which equals to $x(T)$.

\subsection{Proof of Lemma 3}

In the following, we denote the images $\left\{f(t): t \in\left[T_{1}, T_{2}\right]\right\}$ and $\left\{g(t): t \in\left[T_{3}, T_{4}\right]\right\}$ simply by $f$ and $g$, respectively. Since $f$ and $g$ are closed and bounded sets in $\mathbb{R}^{2}$, the intersection $f \cap g$ becomes a compact set. Thus, if the set $f \cap g$ has infinitely many elements, then there exists an accumulation point in $f \cap g$. For a point $\left(t_{0}, s_{0}\right), t_{0} \in\left[T_{1}, T_{2}\right], s_{0} \in\left[T_{3}, T_{4}\right]$, in $f \cap g$, if the direction vectors $\frac{\mathrm{d} f}{\mathrm{~d} t}\left(t_{0}\right)$ and $\frac{\mathrm{d} g}{\mathrm{~d} t}\left(s_{0}\right)$ are parallel, i.e., there exists $\alpha \in \mathbb{R}$ such that $\frac{\mathrm{d} f}{\mathrm{~d} t}\left(t_{0}\right)=\alpha \frac{\mathrm{d} g}{\mathrm{~d} t}\left(s_{0}\right)$, we call $\left(t_{0}, s_{0}\right)$ a tangent point.

We show Lemma 3 by proving that there is no accumulation point in $f \cap g$. We first establish that an accumulation point must be a tangent point as Lemma 4. Then, it will be proved that a tangent point also cannot to be an accumulation point, which is formally stated in Lemma 5. To state the first lemma, define $h: \mathbb{R}^{2} \rightarrow \mathbb{R}^{2}$ by

$$
h(t, s):=\mathrm{e}^{\Lambda t} a+\mathrm{e}^{\Lambda s} b+c .
$$

The following lemma holds.

Lemma 4. Suppose $h\left(t_{0}, s_{0}\right)=\mathbf{0}$ and $\left(t_{0}, s_{0}\right)$ is not a tangent point of $f$ and $g$. Then there exists a neighborhood of $\left(t_{0}, s_{0}\right)$ in which $h(t, s) \neq \mathbf{0}$ holds except at $\left(t_{0}, s_{0}\right)$.

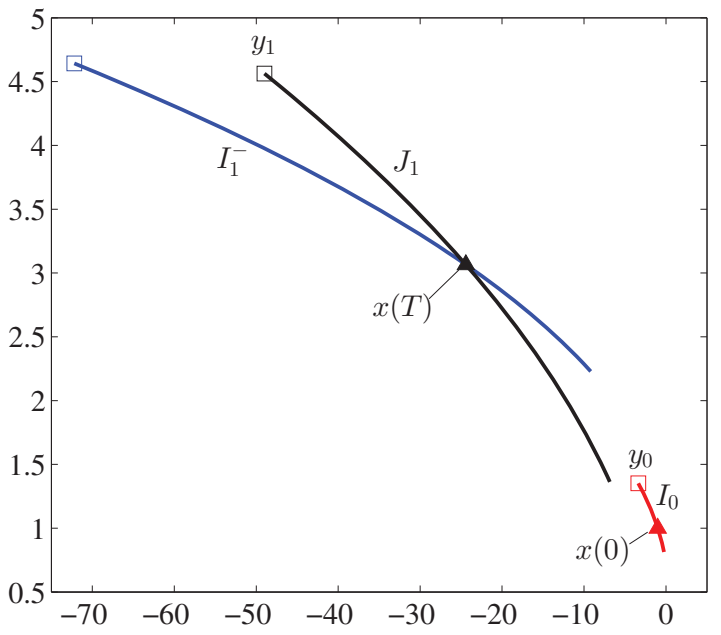

Figure 6: Estimation sets $I_{0}, I_{1}^{-}$, and $J_{1}$ in the state space $\mathbb{R}^{2}$ (horizontal axis: first element of $x$, vertical axis: second element of $x$ ).

Proof. Define $h_{1}$ and $h_{2}$ by

$$
\begin{aligned}
& h_{1}(t):= \begin{cases}\frac{\mathrm{e}^{\Lambda\left(t-t_{0}\right)}-I}{t-t_{0}} \mathrm{e}^{\Lambda t_{0}} a & \text { if } t \neq t_{0}, \\
\Lambda \mathrm{e}^{\Lambda t_{0}} a & \text { if } t=t_{0},\end{cases} \\
& h_{2}(s):= \begin{cases}\frac{\mathrm{e}^{\Lambda\left(s-s_{0}\right)}-I}{s-s_{0}} \mathrm{e}^{\Lambda s_{0}} b & \text { if } s \neq s_{0}, \\
\Lambda \mathrm{e}^{\Lambda s_{0}} b & \text { if } s=s_{0} .\end{cases}
\end{aligned}
$$

Then $h_{1}$ and $h_{2}$ are continuous and it follows that

$$
h(t, s)=\left(t-t_{0}\right) h_{1}(t)+\left(s-s_{0}\right) h_{2}(s) .
$$

We will show that $h_{1}(t)$ and $h_{2}(s)$ are independent in a neighborhood of $\left(t_{0}, s_{0}\right)$ by contradiction. Pick any $\epsilon>0$ and consider the ball $B_{\epsilon}:=\left\{(t, s):\left\|(t, s)-\left(t_{0}, s_{0}\right)\right\|<\right.$ $\epsilon\}$. Assume that there exists $(t, s) \in B_{\epsilon}$ such that for some $\alpha_{t, s} \in \mathbb{R}$

$$
h_{1}(t)=\alpha_{t, s} h_{2}(s) .
$$

From continuity of $h_{1}$ and $h_{2}$, there exist $l_{1}(t)$ and $l_{2}(s)$ such that $h_{1}(t)=h_{1}\left(t_{0}\right)+l_{1}(t), h_{2}(s)=h_{2}\left(s_{0}\right)+l_{2}(s)$, and $\lim _{t \rightarrow t_{0}} l_{1}(t)=\lim _{s \rightarrow s_{0}} l_{2}(s)=\mathbf{0}$. For these vectors, it follows that

$$
\left\|l_{1}(t)-\alpha_{t, s} l_{2}(s)\right\| \leq\left\|l_{1}(t)\right\|+\left|\alpha_{t, s}\right|\left\|l_{2}(s)\right\| .
$$

Here, since $\left\|h_{1}(t)\right\|$ and $\left\|h_{2}(s)\right\|$ are bounded by positive constants respectively from above and below on $B_{\epsilon}$, and by (26), $\left|\alpha_{t, s}\right|$ is bounded from above. Thus, for a sufficiently small $\epsilon$, the right-hand side of the above inequality can be arbitrarily small. On the other hand, with (26), it follows that

$$
\left\|l_{1}(t)-\alpha_{t, s} l_{2}(s)\right\|=\left\|h_{1}\left(t_{0}\right)-\alpha_{t, s} h_{2}\left(s_{0}\right)\right\| .
$$

Since $\left(t_{0}, s_{0}\right)$ is not a tangent point, for any $\alpha \in \mathbb{R}, h_{1}\left(t_{0}\right) \neq$ $\alpha h_{2}\left(s_{0}\right)$. Therefore, the right-hand side of (27) is bounded from below as

$$
\left\|h_{1}\left(t_{0}\right)-\alpha_{t, s} h_{2}\left(s_{0}\right)\right\|>\gamma,
$$


where $\gamma:=\inf _{\alpha \in \mathbb{R}}\left\|h_{1}\left(t_{0}\right)-\alpha h_{2}\left(s_{0}\right)\right\|>0$ is the shortest distance from $h_{1}\left(t_{0}\right)$ to $\left\{\alpha h_{2}\left(s_{0}\right): \alpha \in \mathbb{R}\right\}$. This contradiction shows that if we take a sufficiently small $\epsilon$, then (26) does not hold for any point in $B_{\epsilon}$. That is, $h_{1}(t)$ and $h_{2}(s)$ are linearly independent in $B_{\epsilon}$. Recalling (25), we have that $h(t, s)=\mathbf{0}$ on $B_{\epsilon}$ only at $(t, s)=\left(t_{0}, s_{0}\right)$.

Now we show that a tangent point in $f \cap g$ also cannot to be an accumulation point if we choose $a, b$, or $c$ not to be eigenvectors of $\Lambda$.

Lemma 5. Suppose that

$$
f\left(t_{0}\right)=g\left(s_{0}\right)
$$

and $\left(t_{0}, s_{0}\right)$ is a tangent point. If there exists a sequence $\left\{\left(t_{k}, s_{k}\right)\right\}_{k=1}^{\infty}$ converging to $\left(t_{0}, s_{0}\right)$ and satisfying

$$
f\left(t_{k}\right)=g\left(s_{k}\right), \quad \forall k \in \mathbb{Z}_{+},
$$

then $a, b$, and $c$ are eigenvectors of $\Lambda$.

Proof. Since $\left(t_{0}, s_{0}\right)$ is a tangent point, we have

$$
\mathrm{e}^{\Lambda t_{0}} a=\alpha \mathrm{e}^{\Lambda s_{0}} b
$$

for some constant $\alpha \neq 0$. Moreover, from (28) it follows that

$$
\mathrm{e}^{\Lambda t_{0}} a=\mathrm{e}^{\Lambda s_{0}} b+c .
$$

With (30) and (31), we have

$$
c=(\alpha-1) \mathrm{e}^{\Lambda s_{0}} b .
$$

Since $c$ is nonzero, $\alpha \neq 1$.

We show that there exists no sequence $\left\{\left(u_{k}, v_{k}\right)\right\}_{k=1}^{\infty}$ such that (29) holds with $t_{k}=t_{0}+u_{k}$ and $s_{k}=s_{0}+v_{k}$ and $\left(u_{k}, v_{k}\right)$ goes to $(0,0)$ by contradiction. Suppose $b$ is not an eigenvector of $\Lambda$. Then since $\Lambda=\operatorname{diag}\left(\lambda_{1}, \lambda_{2}\right)$ and $\lambda_{1} \neq \lambda_{2}$, all elements of $b$ are nonzero. From the definitions of $f$ and $g$ and (30), we have

$$
\begin{aligned}
& f\left(t_{k}\right)=f\left(t_{0}+u_{k}\right)=f\left(t_{0}\right)+\alpha\left(\mathrm{e}^{\Lambda u_{k}}-I\right) \mathrm{e}^{\Lambda s_{0}} b, \\
& g\left(s_{k}\right)=g\left(s_{0}+v_{k}\right)=g\left(s_{0}\right)+\left(\mathrm{e}^{\Lambda v_{k}}-I\right) \mathrm{e}^{\Lambda s_{0}} b .
\end{aligned}
$$

Thus by (28) and (29), it follows that

$$
\alpha\left(\mathrm{e}^{\Lambda u_{k}}-I\right) b=\left(\mathrm{e}^{\Lambda v_{k}}-I\right) b .
$$

Since both elements of $b$ are nonzero,

$$
\alpha\left(\mathrm{e}^{\lambda_{i} u_{k}}-1\right)=\mathrm{e}^{\lambda_{i} v_{k}}-1
$$

holds for $i=1,2$. By (33), $v_{k}$ satisfies $v_{k}=v_{\lambda_{i}}\left(u_{k}\right)$ where

$$
v_{\lambda}(u):=\frac{1}{\lambda} \ln \left\{\alpha\left(\mathrm{e}^{\lambda u}-1\right)+1\right\} .
$$

Taking derivative of $v_{\lambda}(u)$, we have

$$
\frac{\mathrm{d} v_{\lambda}}{\mathrm{d} u}=\frac{\alpha \mathrm{e}^{\lambda u}}{\alpha\left(\mathrm{e}^{\lambda u}-1\right)+1}, \quad \frac{\mathrm{d}^{2} v_{\lambda}}{\mathrm{d} u^{2}}=\frac{\alpha \lambda \mathrm{e}^{\lambda u}(1-\alpha)}{\left\{\alpha\left(\mathrm{e}^{\lambda u}-1\right)+1\right\}^{2}} .
$$

Thus $v_{\lambda}(u)$ can be written as

$$
v_{\lambda}(u)=\alpha u+\frac{1}{2} \alpha(1-\alpha) \lambda u^{2}+o\left(u^{2}\right) .
$$

Define $d(u)$ by

$$
\begin{aligned}
d(u) & :=v_{\lambda_{1}}(u)-v_{\lambda_{2}}(u) \\
& =\frac{1}{2} \alpha(1-\alpha)\left(\lambda_{1}-\lambda_{2}\right) u^{2}+o\left(u^{2}\right) .
\end{aligned}
$$

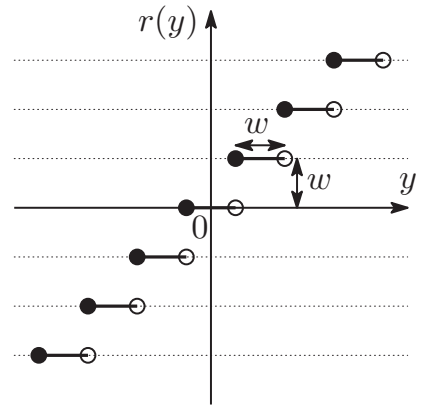

Figure 7: Static uniform quantizer.

We show that $d(u)$ has an isolated zero at $u=0$. Define $\tilde{d}(u)$ as

$$
\tilde{d}(u):= \begin{cases}u^{-2} d(u) & \text { if } u \neq 0, \\ \frac{1}{2} \alpha(1-\alpha)\left(\lambda_{1}-\lambda_{2}\right) & \text { if } u=0 .\end{cases}
$$

Then $\tilde{d}(u)$ is continuous at $u=0$. However,

$$
\tilde{d}(0)=\frac{1}{2} \alpha(1-\alpha)\left(\lambda_{1}-\lambda_{2}\right) \neq 0 .
$$

Therefore, from continuity of $\tilde{d}(u)$, there is a neighborhood of 0 in which $\tilde{d}(u)$ or $d(u)$ has no zero except at $u=0$. This contradicts the existence of the sequences $\left\{\left(u_{k}, v_{k}\right)\right\}_{k}$ or $\left\{\left(t_{k}, s_{k}\right)\right\}_{k}$. Thus, $b$ must be an eigenvector of $\Lambda$.

Finally, from (30) and (32), if $b$ is an eigenvector of $\Lambda$, then $a$ and $c$ are also eigenvectors.

\section{STABILIZATION UNDER CLOCK OFF- SET AND QUANTIZATION}

So far, we have considered that the reported states $y_{k}$ are real values. However, signals in networked control systems are often quantized to discrete values because of the limitation on sensor resolution and restrictions on dealing with signals in digital processors. In this section, we consider a more practical situation and extend the result for the scalar plants case in Section 4. Here, we introduce a quantizer at the sensor side and study that how the two types of imperfection in measurements, quantization errors and clock offsets, affect on the stabilizability of the system.

Consider the scalar system in (14). The plant state is periodically observed and then quantized by the following static and uniform quantizer $r(\cdot)$ :

$$
r(y)=i w, \quad y \in\left[\left(i-\frac{1}{2}\right) w,\left(i+\frac{1}{2}\right) w\right), \quad i \in \mathbb{Z},
$$

where $w>0$ is the width of the quantization cells. In Figure 7 , we show the relation of the input and the output of the quantizer $r(y)$. Then, by (3), the $k$ th reported state $y_{k}$ available at the controller side is represented as

$$
y_{k}:=r\left(x\left(k T+\delta_{k}\right)\right), \quad \delta_{k} \in[0, \Delta] .
$$

The following theorem gives an upper bound on the state achievable under the quantization and the clock offset.

THEOREM 3. If $0 \leq \Delta<\bar{\Delta}$, that is, if the offset bound $\Delta$ satisfies the stability limitation given in Theorem 1, then 


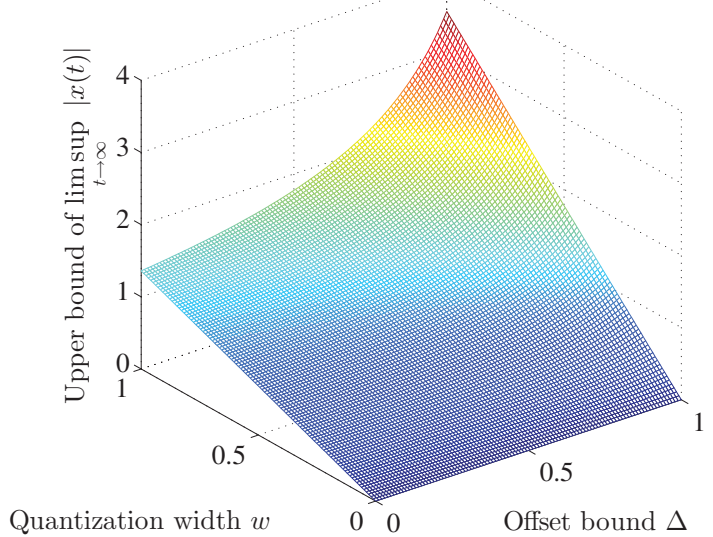

Figure 8: Upper bound of $\limsup _{t \rightarrow \infty}|x(t)|: \lambda=1.0$, $T=1.0$, which is greater than $(\ln 2) / \lambda \approx 0.69$.

there exists a control input $u(t), t \geq 0$, such that

$$
\begin{aligned}
& \limsup _{t \rightarrow \infty}|x(t)| \\
& \leq \begin{cases}\frac{\mathrm{e}^{\lambda T}}{2} w & \text { if } 0<T \leq \frac{1}{\lambda} \ln 2, \\
\frac{\mathrm{e}^{\lambda T}}{2} \frac{w}{\left(\mathrm{e}^{\lambda \Delta}-1\right)\left(1-\mathrm{e}^{\lambda T} / 2\right)+1} & \text { if } T>\frac{1}{\lambda} \ln 2 .\end{cases}
\end{aligned}
$$

We remark that under $0 \leq \Delta<\bar{\Delta}$ and $T>(\ln 2) / \lambda$, the two expressions in (35) satisfy

$$
\frac{\mathrm{e}^{\lambda T}}{2} w<\frac{\mathrm{e}^{\lambda T}}{2} \frac{w}{\left(\mathrm{e}^{\lambda \Delta}-1\right)\left(1-\mathrm{e}^{\lambda T} / 2\right)+1} .
$$

This fact is consistent with one's intuition in which we have tighter upper bound on $\lim \sup _{t \rightarrow \infty}|x(t)|$ for smaller sampling interval $T$. The example below illustrates how the offset bound $\Delta$ and the width of quantization $w$ affect on the upper bound (35).

EXAmPle 4. Consider the scalar system where $\lambda=1.0$ and $T=1.0$. In Figure 8, we have plotted the upper bound on $\lim \sup _{t \rightarrow \infty}|x(t)|$ against the offset bound $\Delta$ and the width of quantization $w$. Note that $T=1.0>(\ln 2) / \lambda \approx$ 0.69 . Hence, the figure represents the second expression in (35). We observe that the bound (35) is monotonically increasing linearly with $w$ and exponentially with $\Delta$.

To show Theorem 3, we follow the approach in the proofs of Theorem 1 and Lemma 1. The main difference for this case is that, due to the imperfection by quantization, the estimation set $J_{k}$ computed from the observation $y_{k}$ now has two uncertain parameters; the offset and the quantization error.

Proof of Theorem 3. With (16) and the definition of the quantizer (34), the estimation set $J_{k}$ based on the quantized observation $y_{k}$ results in

$$
\begin{array}{r}
J_{k}=\left\{\mathrm{e}^{-\lambda \delta_{k}}\left(y_{k}+r_{k}+\lambda^{-1} u_{k}\right)-\lambda^{-1} u_{k}\right. \\
\left.: \delta_{k} \in[0, \Delta], r_{k} \in\left[-\frac{w}{2}, \frac{w}{2}\right]\right\} .
\end{array}
$$

Here, $r_{k}$ is the quantization error in $y_{k}$. By following the proof of Lemma 1, we introduce the notations:

$$
\tilde{y}:=y_{k}+\lambda^{-1} u_{k}, \quad \tilde{J}:=\left\{z+\lambda^{-1} u_{k}: z \in J_{k}\right\} .
$$

From (36), $\tilde{J}$ is expressed as

$$
\tilde{J}= \begin{cases}{\left[\mathrm{e}^{-\lambda \Delta}\left(\tilde{y}-\frac{w}{2}\right), \tilde{y}+\frac{w}{2}\right]} & \text { if } \tilde{y}-\frac{w}{2}>0, \\ {\left[-\frac{w}{2}, \frac{w}{2}\right]} & \text { if }-\frac{w}{2} \leq \tilde{y} \leq \frac{w}{2}, \\ {\left[\tilde{y}-\frac{w}{2}, \mathrm{e}^{-\lambda \Delta}\left(\tilde{y}+\frac{w}{2}\right)\right]} & \text { if } \tilde{y}+\frac{w}{2}<0\end{cases}
$$

Now fix $I_{k}^{-}$and find an upper bound of the length $l_{k}$ of the updated estimation set $I_{k}=I_{k}^{-} \cap J_{k}$. Let $\tilde{I}^{-}:=$ $\left\{z+\lambda^{-1} u_{k}: z \in I_{k}^{-}\right\}=[p, q]$ and $\tilde{I}:=\tilde{I}^{-} \cap \tilde{J}$. In view of the proof of Lemma 1, we take the control input $u(t)$, $t \in((k-1) T+\Delta, k T)$ to make $\tilde{I}^{-}$symmetry about the origin. Under such this input, we have

$$
|q|=|p|=\frac{\mathrm{e}^{\lambda T} l_{k-1}}{2} .
$$

We show that under this control input, the length $l_{k}$ of $I_{k}$ or $\tilde{I}$ is bounded as follows:

$$
\begin{aligned}
& \max _{x_{k} \in I_{k}^{-}, \delta_{k} \in[0, \Delta]} l_{k} \\
& \leq \begin{cases}w & \text { if } l_{k-1} \leq 2 \mathrm{e}^{-\lambda T} w, \\
\left(1-\mathrm{e}^{-\lambda \Delta}\right) \frac{\mathrm{e}^{\lambda T}}{2} l_{k-1}+w \mathrm{e}^{-\lambda \Delta} & \text { else. }\end{cases}
\end{aligned}
$$

From (38), $p<0$ and $q>0$, and hence by (37), we have

$$
\tilde{I}= \begin{cases}{\left[\mathrm{e}^{-\lambda \Delta}\left(\tilde{y}-\frac{w}{2}\right), \min \left(q, \tilde{y}+\frac{w}{2}\right)\right]} & \text { if } \tilde{y} \geq \frac{w}{2} \\ {\left[\max \left(p,-\frac{w}{2}\right), \min \left(q, \frac{w}{2}\right)\right]} & \text { if }-\frac{w}{2} \leq \tilde{y}<\frac{w}{2}, \\ {\left[\max \left(p, \tilde{y}-\frac{w}{2}\right), \mathrm{e}^{-\lambda \Delta}\left(\tilde{y}+\frac{w}{2}\right)\right]} & \text { if } \tilde{y}<-\frac{w}{2}\end{cases}
$$

Therefore,

$$
l_{k} \leq \begin{cases}\left(1-\mathrm{e}^{-\lambda \Delta}\right) q+w \mathrm{e}^{-\lambda \Delta} & \text { if } \tilde{y} \geq \frac{w}{2}, \\ w & \text { if }-\frac{w}{2} \leq \tilde{y}<\frac{w}{2}, \\ -\left(1-\mathrm{e}^{-\lambda \Delta}\right) p+w \mathrm{e}^{-\lambda \Delta} & \text { if } \tilde{y}<-\frac{w}{2} .\end{cases}
$$

Substituting (38) into the above upper bounds yields the following inequality between $l_{k}$ and $l_{k-1}$ :

$$
l_{k} \leq \begin{cases}w & \text { if }-\frac{w}{2} \leq \tilde{y}<\frac{w}{2}, \\ \left(1-\mathrm{e}^{-\lambda \Delta}\right) \frac{\mathrm{e}^{\lambda T}}{2} l_{k-1}+w \mathrm{e}^{-\lambda \Delta} & \text { else. }\end{cases}
$$

Comparing these two upper bounds, we see that

$$
w \geq\left(1-\mathrm{e}^{-\lambda \Delta}\right) \mathrm{e}^{\lambda T} \frac{l_{k-1}}{2}+w \mathrm{e}^{-\lambda \Delta}
$$

is equivalent to $l_{k-1} \leq 2 \mathrm{e}^{-\lambda T} w$. Thus, the inequality (39) holds.

We next consider an upper bound on $\lim \sup _{k \rightarrow \infty} l_{k}$ from (39) and derive the bound (35) on $\lim \sup _{t \rightarrow \infty}|x(t)|$. Define the sequence $\left\{l_{k}^{\prime}\right\}_{k=0}^{\infty}$ by

$$
l_{k+1}^{\prime}:=\left(1-\mathrm{e}^{-\lambda \Delta}\right) \frac{\mathrm{e}^{\lambda T}}{2} l_{k}^{\prime}+w \mathrm{e}^{-\lambda \Delta}, \quad l_{0}^{\prime}:=l_{0} .
$$

Under the condition $0 \leq \Delta<\bar{\Delta}, l_{k}^{\prime}$ converges to

$$
\frac{w}{\left(\mathrm{e}^{\lambda \Delta}-1\right)\left(1-\mathrm{e}^{\lambda T} / 2\right)+1},
$$

and this limit satisfies

$$
w \geq \frac{w}{\left(\mathrm{e}^{\lambda \Delta}-1\right)\left(1-\mathrm{e}^{\lambda T} / 2\right)+1}
$$


for the case $0<T \leq(\ln 2) / \lambda$. Hence, by (39), it follows that

$$
\limsup _{k \rightarrow \infty} l_{k} \leq \begin{cases}w & \text { if } 0<T \leq \frac{1}{\lambda} \ln 2, \\ \frac{w}{\left(\mathrm{e}^{\lambda \Delta}-1\right)\left(1-\mathrm{e}^{\lambda T} / 2\right)+1} & \text { if } T>\frac{1}{\lambda} \ln 2,\end{cases}
$$

for any $x(0)$ and $\delta_{k} \in[0, \Delta], k \in \mathbb{Z}_{+}$. Finally, for all $t \in$ $[k T,(k+1) T], k \in \mathbb{Z}_{+}$, the plant state is bounded as

$$
|x(t)| \leq \mathrm{e}^{\lambda T}|x(k T)| \leq \mathrm{e}^{\lambda T} \frac{l_{k}}{2} .
$$

This fact and (40) conclude the theorem.

\section{CONCLUSION}

This paper has studied stabilization of a linear system using an asynchronous pair of a sensor and a controller. We have derived conditions on the clock offset for stabilization of the system with a single controller. The condition in the scalar plants case gives a tight limitation on the offset, which depends on the level of instability of the plant and the sampling period. For the vector plants case, we have shown that if the plant has at least two different real poles, then the system is stabilizable in finite steps for any large offsets. Although a stabilizing algorithm has been given in the proof, it is based on controllability gramians and is not very suitable for practical controllers. It would be an interesting topic left for future work to study practical control algorithms. Another topic is the investigation of quantization effects for the vector plants case. In this paper, quantization has been considered in the scalar plants case. The main challenge in the vector case is to evaluate the volume of the intersection of $I_{k}^{-}$and $J_{k}$, which result in bands of exponential curves in $\mathbb{R}^{n}$. We also would like to consider more practical situations including control under noises and uncertainty in the plant dynamics.

Acknowledgment: The authors would like to thank Prof. Kenji Kashima at Kyoto University for the proof of Lemma 5

\section{REFERENCES}

[1] P. Barooah and J. P. Hespanha. Estimation on graphs from relative measurements. IEEE Control Syst. Mag., 7(4):57-74, 2007.

[2] C. Bonebrake and L. R. O'Neil. Attacks on GPS time reliability. IEEE Security Privacy, 12(3):82-84, 2014.

[3] A. Cervin, D. Henriksson, B. Lincoln, J. Eker, and K.-E. Årzén. How does control timing affect performance? Analysis and simulation of timing using Jitterbug and TrueTime. IEEE Control Syst. Mag., 23(3):16-30, 2003.

[4] R. I. Davis and A. Burns. A survey of hard real-time scheduling for multiprocessor systems. $A C M$ Computing Surveys, 43(4):1-44, 2011.

[5] M. C. F. Donkers, W. P. M. H. Heemels, N. van de Wouw, and L. Hetel. Stability analysis of networked control systems using a switched linear systems approach. IEEE Trans. Autom. Control, 56(9):2101-2115, 2011.

[6] F. Dörfler and F. Bullo. Synchronization in complex networks of phase oscillators: A survey. Automatica, 50(6):1539-1564, 2014.
[7] N. M. Freris, S. R. Graham, and P. R. Kumar. Fundamental limits on synchronizing clocks over networks. IEEE Trans. Autom. Control, 56(6):1352-1364, 2011.

[8] H. Fujioka. Stability analysis of systems with aperiodic sample-and-hold devices. Automatica, 45(3):771-775, 2009.

[9] J. P. Hespanha, P. Naghshtabrizi, and Y. Xu. A survey of recent results in networked control systems. Proc. IEEE, 95(1):138-172, 2007.

[10] IEEE Std 1588-2008, IEEE Standard for a Precision Clock Synchronization Protocol for Networked Measurement and Control Systems. IEEE Instrumentation and Measurement Society, 2008.

[11] X. Jiang, J. Zhang, B. J. Harding, J. J. Makela, and A. D. Dominguez-Garcia. Spoofing GPS receiver clock offset of phasor measurement units. IEEE Trans. Power Syst., 28(3):3253-3262, 2013.

[12] H. Kopetz. Real-Time Systems. Springer, New York, USA, 2nd ed., 2011.

[13] L. A. Montestruque and P. Antsaklis. Stability of model-based networked control systems with time-varying transmission times. IEEE Trans. Autom. Control, 49(9):1562-1572, 2004.

[14] P. Naghshtabrizi, J. P. Hespanha, and A. R. Teel. Stability of delay impulsive systems with application to networked control systems. Trans. Inst. Measurement Control, 32(5):511-528, 2010.

[15] G. N. Nair and R. J. Evans. Stabilizability of stochastic linear systems with finite feedback data rates. SIAM J. Control Optim., 43(2):413-436, 2004.

[16] G. N. Nair, F. Fagnani, S. Zampieri, and R. J. Evans. Feedback control under data rate constraints: An overview. Proc. IEEE, 95(1):108-137, 2007.

[17] L. Sha, T. Abdelzaher, K.-E. Årzén, A. Cervin, T. Baker, A. Burns, G. Buttazzo, M. Caccamo, J. Lehoczky, and A. K. Mok. Real time scheduling theory: A historical perspective. Real-Time Systems, 28(2-3):101-155, 2004.

[18] B. Sundararaman, U. Buy, and A. D. Kshemkalyani. Clock synchronization for wireless sensor networks: A survey. Ad Hoc Networks, 3(3):281-323, 2005.

[19] S. Tatikonda and S. Mitter. Control under communication constraints. IEEE Trans. Autom. Control, 49(7):1056-1068, 2004.

[20] M. Wakaiki, K. Okano, and J. P. Hespanha. Stabilization of networked control systems with clock offset. In Proc. American Control Conf., 2015 (to appear).

[21] Y.-C. Wu, Q. Chaudhari, and E. Serpedin. Clock synchronization of wireless sensor networks. IEEE Signal Process. Mag., 28(1):124-138, 2011.

[22] T.-C. Yang. Networked control system: A brief survey. IEE Proc.-Control Theory Appl., 153(4):403-412, 2006 . 